\title{
III. Transactions of the American Otological Society.
}

Forty-seventh annual Meeting.

Atlantic City, N. J. May 27. and 28, 1914. Volume XIII. Part II.

1. Lutz (Brooklyn, N. Y.): Wie in Fällen von chronischer Otitis media catarrhalis der Kranke sich selbst helfen kann. L. erinnert an die mannigfachen Ursachen des chronischen Mittelohrkatarrhs und legt insbesondere die Art und Weise dar, wie Erkrankungen der Nase, ihrer Nebenhöhlen und des Nasenrachenraumes auf Tuba und Paukenhöhle zurückwirken können. Um die durch die Behandlung erreichten Resultate sicherzustellen, ist es notwendig, dem Patienten genaue Ratsehläge für sein weiteres diätetisches Verhalten mitzugeben. Vor allem muß er darüber belehrt werden, wie er einer Verlegung der Nase durch Schleimmassen abzuhelfen, mit anderen Worten, wie er sich in rationeller Weise zu schneuzen hat. Denn die überwiegende Mehrzahl der eitrigen Mittelohrentzündungen läßt sich auf ungeeignete Verfahren zur Reinigung der Nase zurückführen. Der Kranke soll beim Schneuzen sein Taschentuch etwa einen Zoll entfernt von der Nase halten, beide Nasenlöchar sollen frei bleiben und gleichzeitig ausgeblasen werden, die das Tuch haltenden Finger dürfen die Seitenwände der Nase nicht berühren, sondern sind jederseits mindestens einen Zoll entfernt von ihnen anzulegen. Sobald die Luft frei durch die Nase hindurchstreicht, ist mit dem Ausblasen aufzuhören. Gleichzeitiges leichtes Öffnen des Mundes macht jede Druckwirkung auf die Tuben unmöglich.

\section{Diskussion.}

Braislin (Brooklyn) bemerkt, daß ein ungenügender Erfolg der Adenotomie oft in einer zurückgebliebenen Entwicklung der Nase ihre Ursache hat, und daß ein rationelles Ausblasen 
der Nase sowohl hiergegen als prophylaktisch gegen die Bildung adenoider Wucherungen sich nützlich erweist.

Dench (New York) sieht den Grund vieler Mißerfolge in dem Zurücklassen oder Wiederwachsen eines supratonsillaren Lappons.

Myles (New York) hat wiederholt progressive Schwerhörigkeit verursacht durch das Aufwärtswachsen einer tief eingebetteten Gaumentonsille gesehen und wesentliche Besserung nach deren Entfernung.

Barnhill (Indianopolis) hält eine ausreichende Reinigung der Nase in der vom Vortr. angegebenen Weise für unmöglich. Eine mangelhafte Atmung trotz der Adenotomie kann auch durch Deformitäten des Knochengerüstes, wie einen hohen Gaumen, Verbiegungen der Halswirbelsäule oder solehe der Teile innerhalb der Nase bedingt sein. Ein hoher Gaumen müßte nötigenfalls in geeigneter Weise korrigiert werden.

Shurly (Detroit) betont gleich dem Vortr. die Wichtigkeit einer Prophylaxe der Ohrenkrankheiten.

Loeb (St. Louis) hat adenoide Vegetationen trotz vollständiger Beseitigung rezidivieren gesehen.

Sheppard (Brooklyn) sprichi sich zugunsten der vom Vortr. empfohlenen Methode des Schneuzens aus.

Shambaugh (Chicago) glaubt, daß die in Amerika zunehmende Neigung zu Erkältungen durch den Aufenthalt tags und nachts in Räumen, die durch Dampfheizung überhitzt und ausgetrocknet sind, verursacht wird. Er rät behufs Abhärtung zu kühlen Wohnzimmern und im Sommer und Winter ungeheizten und nach außen offenen Schlafzimmern und zu kalten Bädern.

Lewis (New York) spricht sich gleichfalls für die Möglichkeit des Wiederwachsens von adenoiden Vegetationen aus.

Bryan (Washington) führt die von ihm gesehenen Rezidive nach der Adenotomie darauf zurück, daß eine restlose Entfernung des adenoiden Gewebes nicht möglich ist, ohne bis auf den Knochen zu gehen und dadurch Narbenbildungen zu erzeugen, welche mehr als die Vegetationen stören würden. Man soll hierin auch bei ungenügendem Erfolg nicht zu rigoros verfahren. Ferner kann die fortdauernde Wucherung in dem übrigen adenoiden Ringe den Erfolg der Operation beeinträchtigen.

Sheppard erwähnt von ihm beobachtete Fälle, in denen das Wiederwachsen des adenoiden Gewebes, welches wegen ungeeigneten Verhaltens schon begonnen hatte, durch passende 
Diät und sonstige hygienische Maßnahmen zum Rückgang gebracht worden ist.

Barnhill stimmt mit Bryan hinsichtlich der Unmöglichkeit der radikalen Entfernung adenoider Vegetationen überein und erklärt dadurch die häufigen Rezidive.

Loeb glaubt, daß die Rezidive nicht vom zurückgelassenen Gewebe der Vegetationen ausgehen, sondern von einer Neuwucherung des adenoiden Gewebes, das durch den ganzen Pharynxraum verbreitet ist.

Myles gibt zu bedenken, daß bei Kindern unter 2 bis 3 Jahren viele adenoide Follikel noch nicht hypertrophiert sind, später sich aber zu Seiten der Rosenmüllerschen Grube vergrößern, mag operiert worden sein oder nicht. Hiergegen läBt sich nichts tun, ebensowenig wie gegen die Wucherung der Zungentonsille nach Entfernung der Rachenmandel.

Lutz führt in seinem Schlußwort $u$. a. aus, daß auch die akuten Exantheme, die mit entziundlichen Vorgängen im Nasopharynx einhergehen, of das dort vorhandene Lymphgewebe zum Wuchern bringen und dadurch ein Rezidiv der entfernten adenoiden Vegetationen vortäuschen.

2. Bryant (New York). Die konservative und modifizierte Radikaloperation als Mittel, um das Ohr für das Tragen einer Prothese bei herabgesetztem Gehör nach Mittelohreiterung tolerant zu machen. In Fällen, in denen eine chronische Otitis media purulenta mit Zurückbleiben einer beträchtlichen Schwerhörigkeit ausgeheilt ist, das Gehör durch ein künstliches Trommelfell sehr wesentlich gebessert wird, aber die Eiterung bei dessen Tragen regelmäßig wiederkehrt, empfiehlt $\mathrm{Br}$. die genannten Operationen, um ohne Hörverschlechterung durch sie zu bewirken, daß das Ohr die Prothese jetzt verträgt. Die betreffenden Fälle sind gekennzeichnet durch Buchten mit abgestoßenem Epithel, Narbenstränge, Wulstbildungen und die UUberbleibsel der Gehörknöchelchen in Paukenhöhle und Antrum. Der vorzunehmende operative Eingriff richtet sich nach den Verhältnissen im einzelnen Falle. Bei der konservativen Radikaloperation wird in gewöhnlicher Weise die hintere und obere knöcherne Gehörgangswand abgetragen, Attikus, Antrum und Paukenhöhle werden freigelegt, und durch Entfernung der sie ausfüllenden narbigen Massen und Gehörknöchelchenreste wird aus ihnen eine einheitliche und möglichst glattwandige Höhle geschaffen. Dagegen wird Sorge getragen 
füx Erhaltung der Schleimhaut an allen Teilen, die man nicht entfernen will. Die Plastik zielt auf Bildung eines weiten Gehörganges hin. Bei der modifizierten Radikaloperation verfährt man in gleicher Weise, nur daß der Trommelfellrest, sowie Hammer und $A m b o B$ oder, was von ihnen übrig ist, geschont werden. Br. berichtet über zwei Fälle, die in der erwähnten Art, der eine mit Entfernung von Hammer, Amboß und Teilen vom Kopf und den Schenkeln des Steigbügels, der andere mit Erhaltung der Knöchelchenreste, operiert worden sind. Die durch das künstliche Trommelfell zu erzielende Hörverbesserung war eine sehr bedeutende, im ersten Falle von 15 auf $96^{\prime \prime}$ für Politzers Hörmesser. Ob die Paukenhöhlenschleimhaut in der Folge die Prothese reaktionslos vertragen hat, wird nicht gesagt.

\section{Diskussion.}

Dench (New York) erklärt es für überflüssig und sogar schädlich, in jedem Falle das Kürettement der Mittelohrschleimhaut vorzunehmen. Bei einem Kranken, der mit radikaloperiertem einem Ohre und einem Cholesteatom im Recessus epitympanicus der anderen Seite zu ihm kam, hat er die Operation hierselbst auf den oberen Paukenhöhlenraum beschränkt und dadurch dem Manne ein relativ gutes Gehör erhalten.

3. Sheppard (Brooklyn). Die Bedeutung des gestörten "Metabolismus" in der Ätiologie sekretorischer Mittelohrerkrankungen. Nach der Ansicht des Vortr. sind viele Fälle von akutem Tubenkatarrh und von Otitis media mit serösem, schleimigem oder schleimig-eitrigem Sekret in vasomotorischen Lähmungszuständen im Bereiche der Nase, des Nasenrachenraums, der Tuba und der Paukenhöhle begründet, die ihrerseits wieder von einer allgemeinen Toxämie durch gestörten "Metabolismus" - d. i. die Fähigkeit des Organismus, seine Bestandteile fortdauernd aufzubrauchen und zu erneuern - abhängen. Die Ursache dieser Störung liegt entweder in einer fehlerhaften Zufuhr oder einer unzureichenden Ausscheidung durch Darm, Haut und Nieren. Um ersterer Fehlerquelle zu begegnen, muß dafür gesorgt werden, daß die betreffenden Kranken weder im allgemeinen noch von den einzelnen Nahrungsstoffen (Eiweißkörper, Kohlehydrate) zuviel genießen, die Speisen sollen gehörig gekaut werden, bei den Mahlzeiten soll möglichst gar nicht, zwischen ihnen möglichst viel getrunken werden, am besten

Archiv f. Ohrenheilkunde. Bd. 97, Otolog. Rundschau. 
Wasser, während übermäßiger Milchgenuß, starker Kaffee oder Tee, sowie die regelmäßige Aufnahme selbst kleiner Alkoholmengen zu verbieten sind. Der Arzt soll die Diätvorschriften genau bestimmen, da ohne ihre Befolgung eine örtliche Behandlung kaum von Nutzen ist.

\section{Diskussion.}

Lutz -(Brooklyn) stimmt den Ausführungen des Vortr. bei. Er streicht besonders Butter, Milch und Fleisch aus dem Diätzettel,

Me Cuen Smith (Philadelphia) hat nichtinfektiöse Flüssigkeitsansammlungen (Hydrops ex vacuo) in der Paukenhöhle oft bei Frauen gesehen, welche rauchten. Es ist ihm vielfach gelungen, die Ohrgeräusche und das Gefühl von Vollsein, worüber geklagt wurde, durch diätetische Vorschriften zum Schwinden zu bringen.

Shurly (Detroit) hält es für richtig, daß etwa vorhandene Digestionsstörungen wohl beachtet werden müssen, doch warnt er andererseits vor Ưbertreibungen.

Myles (New York) hat gleichfalls durch geeignete Diät und Hygiene sehr gute Erfolge bei subjektiven Gehörsempfindungen gesehen.

Dench (New York) weist auf den von Lermoyez hervorgehobenen übermäßigen Kochsalzgenuß als Krankheitsursache hin. Er hat bei zwei Patienten mit subjektiven Geräuschen (das eine Mal nach Überarbeitung) dadurch Besserung erzielt, daß er bei den Mahlzeiten kleine Mengen von Alkohol nehmen ließ. Die Individualität des Kranken muß berücksichtigt werden, je nachdem er eine größere oder geringere Nahrungszufuhr nötig hat.

Sheppard (SchluBwort) führt die Erfolge bei Ohrensausen durch Alkohol, die auch er beobachtet hat, auf Änderungen im Blutdruck zurück. Seine Patienten waren sowohl Erwachsene als junge Kinder, und zwar lag bei letzteren die Schädlichkeit meist in der überwiegenden Einfuhr von Kohlehydraten.

4. Barnhill (Indianopolis): Zwei Fälle von Sarkom der Dura mater mit dem Ursprung in der Nachbarschaft des Warzenfortsatzes und mit unbestimmten, eine Mastoiditis vortäuschenden Symptomen. Operation, später tödlicher Ausgang. In der ersten Beobachtung, bei einer 36jährigen Frau, bestanden die Symptome in vieljährigen Schmerzen im rechten 
Ohre und der rechten Gesichtshälfte, die sich in der letzten Zeit immer mehr gesteigert hatten. Die Untersuchung ergab Anämie und herabgesetzten Kräftezustand, Strabismus des rechten Auges, leichte Facialisparese, etwas Empfindlichkeit über der rechten Seite des Gesichts und bei tiefem Druck auf den Warzenfortsatz, Taubheit rechts (seit 10 Jahren) mit aufgehobener Knochenleitung, normales Trommelfellbild. Bei der Aufmeißelung wegen der unerträglich gewordenen. Schmerzen zeigte sich der Warzenknochen erweicht und etwa $1^{\text {" }}$ hinter dem Gehörgang von Granulationen durchbrochen. Die weitere Operation deckte einen großen Tumor auf, der die ganze zellige Umgebung nach hinten, innen und oben einnahm und von der Dura mater über und hinter dem Sinus sigmoideus und über der hinteren unteren Partie des Schläfenlappens ausging. Bei der Entfernung der Geschwulst starke Blutung durch Einreißen des Sinus. Nach der Operation Schmerzen bedeutend ermäßigt, aber nicht ganz versehwunden, Heilung der Wunde in einem Monat. Später sollen die Schmerzen in alter Heftigkeit wiedergekehrt und die Kranke, etwa 7 Monate nach dem Eingriff, unter den Zeichen einer Hirnaffektion gestorben sein. Der entfernte Tumor erwies sich als ein Mittelding zwischen Endotheliom und Sarkom. Im zweiten Falle (28jähriger Mann) waren sehr starke Schmerzen im Ohr und Warzenfortsatz seit Wochen vorhanden gewesen und war wegen vermuteter Mastoiditis von anderer Seite die Aufmeißelung gemacht worden. Kein Nachlaß der Schmerzen, Abmagerung, die Operationswunde heilte nicht. B. fand bei seiner Untersuchung, daß aus der Wunde in der Warzengegend eine weiche Masse hervorquoll, ähnlich einem Hirnvorfall, aber in Wirklichkeit sarkomatöser Natur. Die umgebende Haut war stark infiltriert, die benachbarten Lymphdrüsen waren mäBig geschwollen und empfindlich, es bestand reichliche dünnflüssige Eiterung. Trotz gründlicher Entfernung des Tumors, der der Dura mater fest aufsaß, erfolgte schnell ein Wiederwachsen, und der Tod trat 3 Wochen nachher durch Meningitis ein. - B. teilt noch eine dritte Beobachtung mit, betreffend einen 55jährigen Mann mit alter spärlicher Ohreiterung rechts und sich anschließender Thrombose des Sinus sigmoideus und der Ven. jugularis. Die Symptome hatten hauptsächlich in sehr starken Kopfschmerzen, besonders in der Schläfengegend, aber niemals im Ohre oder dessen Umgebung, bestanden, die Temperatur war lange Zeit nur wenig erhöht $\left(37,8^{\circ}\right)$, erst in den letzten Tagen 
zeigte sich leichtes Frösteln. Trotzdem wurde bei der Operation der ganze Sinus sigmoideus bis in das Torcular hinein und ebenso die Jugularis in ihrem oberen Teile mit Eiter erfült gefunden. Breite Eröffnung des Sinus, Unterbindung der Vene dicht über der Clavicula. Abfall des Fiebers und Nachlaß der Schmerzen, dann aber nach 10 Tagen neuer Anstieg, septische Temperaturen und rechtsseitiger Exophthalmus als Zeichen einer Cavernosusthrombose. Der Kranke reiste in seine Heimat zurüick. Dort trat eine auffällige Besserung ein, Fieber und Exophthalmus verschwanden, der Kräftezustand hob sich, Patient konnte umhergehen, starb aber ganz plötzlich wahrscheinlich infolge einer Embolie.

\section{Diskussion.}

Lutz (Brooklyn) und Bryant (New York) erwähnen kurz drei von ihnen beobachtete Sarkom- bzw. Endotheliomfälle.

Day (Pittsburg) bezweifelt die Cavernosusthrombose in dem dritten Falle des Vortr. wegen des nur rechtsseitigen Exophthalmus, da er nicht glaubt, daß der eine Sinus cavernosus thrombosieren kann, ohne daß sich auch der andere daran beteiligt. Protrusio bulbi kann auch aus anderen Ursachen entstehen. So hat D. einen Knaben mit Infektion nach einer Verletzung und beiderseitigem Exophthalmus gesehen, bei dessen Sektion sich herausstellte, daß der stark erhöhte Hirndruck eine vollständige Obliteration beider Sinus cavernosi bewirkt hatte. In einem anderen Falle machte er eine Operation behufs Druckentlastung des Schädelinhalts, spaltete die Dura und konstatierte eine Ruptur des dritten Ventrikels. Unter einer 3 Monate hindurch aufrecht erhaltenen Dränage verschwand der Exophthalmus zugleich mit den anderen Symptomen.

Mc Cuen Smith (Philadelphia) hält die Diagnose einer Cavernosusthrombose ohne Bestätigung durch die Sektion für nicht sicher. Die von ihm beobachteten Fälle sind sämtlich mit dem Tode ausgegangen.

Crockett (Boston) berichtet über «wei gute Erfolge von der Behandlung eines Ohrsarkoms mit Coleyscher Flüssigkeit. In einem dritten Falle von Sarkom (wo ?) mit Exophthalmus hat er $20 \mathrm{mg}$ Radium in das Orbitalfett eingelegt und danach vollständige Heilung erhalten; es hinterblieb nur eine kleine lineare Narbe, wie nach der Killianschen Operation.

Dench (New York) hat drei Fälle von Ohrsarkom operiert, zwei bei Erwachsenen und einen bei einem Kinde. Von diesen 
sind zwei gestorben, der dritte ist geheilt worden. $\operatorname{Er}$ bestätigt die Gesichtsschmerzen als Symptom der Krankheit. Bei einer Cavernosusthrombose würde er die von Ballance vorgeschlagene Operation versuchen.

Braislin (Brooklyn) hat bei einem Sarkom der Mandel Heilung durch die Coleysche Flüssigkeit gesehen.

Barnhill (Schlußwort) erwidert, daß in seinem Falle alle Symptome für eine Thrombose des Sinus cavernosus sprachen. Er ist bei seinen sonstigen iiblen Erfahrungen durch die spontane Rückbildung des Leidens selbst höchst überrascht worden. Das Verfahren von Ballance, um den Sinus cavernosus zugänglich zu machen, läßt sich bei starker Kongestion der Teile wohl kaum ausführen.

Day bemerkt hierzu, daß man durch vorherige Ventrikelpunktion genügend Raum gewinnen könnte, doch würde in 3 bis 4 Tagen eine Meningitis nachfolgen.

5. Braislin (Brooklyn). Weitere Bemerkungen über die Applikation von Argentum nitricum auf das Ostium pharyngeum tubae zur Beseitigung von Ohrensausen. Br. ergänzt seine früheren Angaben über diesen Gegenstand (vergl. dieses Archiv, Bd. 91, S. 104). Den biegsamen Draht, an dessen Ende in näher beschriebener Weise das Wattestückchen befestigt wird, stellt er aus zwei fest zusammengedrehten Silberfäden her, die Höllensteinlösung nimmt er gewöhnlich in Stärke von 4 Proz., aber auch konzentrierter, wenn die Applikation frühestens nach einer Woche wiederholt wird. Die Betupfung geschieht nach beendigter Luftdusche. Als hauptsächlichstes Indikationsgebiet für diese Behandlung bezeichnet Br. die Fälle von subjektiven Geräuschen, die mit Kongestion und Schwellung der Tubenschleimhaut verbunden sind, während sie bei Ohrensausen durch Erkrankung des Akustikus oder aus zentraler Ursache wirkungslos bleibt. Die Höllensteinbehandlung muß mit der sonst durch den Fall angezeigten lokalen Therapie in $\mathrm{Ohr}$ und Nase und mit der erforderlichen Rücksichtnahme auf etwaige Digestions-, Zirkulations- oder Allgemeinstörungen verbunden werden.

\section{Diskussion.}

Dench (New York) rät, anstatt des Silberdrahtes ein Stück einer Klaviersaite zu nehmen, an deren vorderem, zu einem kleinen Häkchen umgebogenen Ende man die Watte befestigt. 
8. Crockett (Boston): Entfernung des Steigbügels zum Zwecke der Heilung von starkem Ohrschwindel. Cr. berichtet über die Nachgeschichte verschiedener, bereits veröffentlichter, von ihm mit Stapesextraktion behandelter Fälle. - a) Schwindel von wechselnder Intensität, zeitweise stark und kontinuierlich, manchmal in apoplektiformen Anfällen mit Hinstürzen. Beide Trommelfelle verdickt, eingezogen, mit Verkalkungen. Uhr links $37 / 60$, Sprache 20/25, rechts Uhr $4 / 60$, Sprache $2 / 25$, untere Tongrenze 128 v. s. Katheterismus und Amboßextraktion ohne Nutzen. Entfernung des Steigbügels, der frei beweglich war. Patient lebte noch 12 Jahre, in denen er nur noch einmal 2-3 Monate lang leichten Schwindel hatte. Gehör rechts nach der Operation völlig erloschen. - b) Andauernder heftiger Schwindel, der den Kranken arbeitsunfähig machte. stark eingezogene, narbig veränderte Trommelfelle. Gehör rechts normal, links Uhr 2/60, Sprache 2/25, Knochenleitung verkürzt, untere Tongrenze 512 v. s., obere Tongrenze normal. Nach wirkungslos gebliebener sonstiger Behandlung Extraktion des linken Steigbügels. Seitdem (12 Jahre) kein Schwindel mehr. 2 Wochen nach der Operation arbeitsfähig. Totale Taubheit des operierten Ohres. - c) In früheren Jahren ausgesprochene Ménièresche Anfälle, in der letzten Zeit starker kontinuierlicher Schwindel, schwankender Gang, Gefühl von Völle im Kopf, psychische Depression, oft täglich 1-3 mal Übelkeit und Erbrechen. Trommelfelle stark verdickt, rechts normales Gehör, links lautes Uhrticken 2", laute Sprache 6", untere Tongrenze $512 \mathrm{v}, \mathrm{s}$. , obere Tongrenze normal. Extraktion des (frei beweglichen) Steigbügels. Patient verließ die Klinik nach 3 Tagen und war nach einer Woche arbeitsfähig. In den nächsten Monaten hatte er noch wiederholt ganz leichten Schwindel von 2-3 Minuten Dauer, aber ohne Erbrechen oder Übelkeit, und war sein Gang noch etwas unsicher, seit 4 oder 5 Jahren ist aber auch dieses verschwunden. Das operierte Ohr ist taub. - d) Seit 6 Jahren Schwindelanfälle, früher apoplektiform, in letzter Zeit leichterer Art, durch ihre große Häufigkeit aber höchst störend. Trommelfelle normal, ebenso das Gehör auf der linken Seite, rechts Uhr 2/80, Sprache 0, untere Tongrenze 96 v. s. Entfernung des Steigbügels, dabei Facialiszucken. Schwindel nicht wiedergekehrt, totale Taubheit rechts, vollständige Facialislähmung. - e) Alter Mann mit Arteriosklerose und Schwindelanfällen, die seit der Stapesextraktion vor einem Jahre nicht wieder- 
gekehrt sind. - Cr. ist der Ansicht, daß die bloße Entfernung des Steigbügels bei unerträglichem Schwindel und Ohrensausen die weit eingreifendere Labyrinthoperation ersetzen kann. Doch sollte sie nur nach einer sorgfältigen Analyse der Symptome, dem Ausschluß einer Erkrankung des Zentralnervensystems und nach vorheriger, unwirksam gebliebener Mittelohrbehandlung vorgenommen werden. Ein Erfolg läßt sich nur erwarten, wenn die nachfolgende Entzündung die Labyrinthfunktionen vollständig aufhebt, also das operierte Ohr total taub wird.

\section{Diskussion.}

Barnhill (Indianopolis) fragt den Vortr., auf welche Weise er sich bei doppelseitiger Erkrankung über das zu operierende $\mathrm{Ohr}$ entscheidet.

Dench (New York) hat in einigen Fällen vollständiges Aufhören des Schwindels durch Einspritzung von 2 Tropfen einer 2 prozentigen Pilokarpinlösung durch den Katheter in die Paukenhöhle erreicht. Diese Behandlung kann sich auch da nützlich erweisen, wo die innerliche Darreichung von Pilokarpin ohne Erfolg geblieben ist.

Crockett (Schlußwort) erwidert, daB er in den ersten 3 Fällen vor der Operation auch die Pilokarpineinspritzung versucht hat. Zur Zeit, als die heutigen Methoden der Labyrinthuntersuchung noch nicht bekannt waren, hat er den Eingriff stets an dem schlechter hörenden Ohre gemacht, jetzt würde er natürlich die Wahl nach den Ergebnissen der Prüfung der Vestibularfunktion treffen.

7. Dench (New York): Über die Behandlung von zufälligen Verletzungen der Dura mater bei den Mastoidoperationen. Verletzungen der erwähnten Art scheinen nicht gerade häufig zu sein und kommen ebensowohl an einer vollständig gesunden als schon vorher erkrankten, nekrotischen Dura vor. Unter den 6 mitgeteilten Beobachtangen war letzteres dreimal der Fall. Die Duraverletzung machte bei 3 Kranken gar keine Folgeerscheinungen, einmal zeigte sich unmittelbar nachher eine leichte Temperaturerhöhung (bis höchstens $38^{\circ}$ ) und etwas Kopfschmerz, einmal (Verletzung der Dura durch einen Knochensplitter) entstand eine ganz umschriebene oberflächliche Hirnnekrose, ein Patient mit akuter Mastoiditis ging an einer (wahrscheinlich schon vorher eingeleiteten) Meningitis zugrunde. Zur Verhütung übler Folgen einer etwa eintretenden Verletzung der Dura 
ist vor allem peinlichste Asepsis bei der Operation notwendig. Ist eine Verletzung geschehen, so soll man die Durawunde mit Jodoformgaze bedecken und dann die Operation weiter fortführen, wobei besondere Sorge dafür getragen werden muß, daB nirgends ein Eiterherd zurückbleibt, und daß die Durawunde durch ausgiebige Entfernung des Knochens in ihrer Umgebung übersichtlich freiliegt. Die Durawunde wird durch einen Kreuzschnitt, erweitert und die Öffnung ziemlich fest mit Jodoformgaze ausgefüllt, um durch den Druck eine Verklebung der Meningen ringsumher zu erzielen. Wenn sich eine akute Encephalitis entwickelt, woran man immer denken muß, beugt man durch eine freie Dränage des infizierten Bezirkes am besten dem Weiterumsichgreifen vor.

\section{Diskussion.}

(Rae New York) meint, daß von einem aseptischen Operieren in einer eiternden Höhle nicht die Rede sein kann. Duraverletzungen während der Mastoidoperation sind unter Umständen unvermeidlich. Sie sind gefährlicher in akuten als in chronischen Fällen, bei denen durch die bereits eingetretene Reaktionstätigkeit der Gewebe dem Fortschreiten der Infekion schon entgegengearbeitet worden ist.

Mc Cuen Smith (Philadelphia) häli eine gesunde Dura für widerstandsfähig genug, um auch gröberen Insulten widerstehen zu können. In den betreffenden Fällen war die Dura wahrscheinlich immer sehon, wenn auch noch nieht wahrnehmbar, erkrankt. Er hat die vom Vortr. gegebenen Ratschläge schon seit Jahren befolgt und legt das Hauptgewicht auf die freie Dränage.

Dench (Schlußwort) betont, daß in 3 von seinen Fällen die Dura ganz gesund gewesen ist. Anstatt, wie Day empfohlen hat, einen Druck auf die Dura rings um die Wundränder auszuüben, fübrt er die Gaze zwischen die Wundränder ein, weil or glaubt, auf dieso Weise die Schichten der Pia und den Subduralraum am besten zum Abschluß zu bringen.

8. Mosher (Boston): Die Annäherung an den Sinus cavernosus durch die Orbita. Die Operation besteht ihrem Wesen nach in einer Erweiterung der Fissura orbitalis superior durch Fortnahme des sie nach unten begrenzenden inneren Teiles des großen Keilbeinflügels. Nachdem der (in solchen Fällen regelnäBig schon erblindete) Augapfel entfernt und die Orbita ausgeräumt worden ist, wird zunächst 
von der hinteren Hälfte ihres Bodens das Periost abgeschabt und die Rinne, in der der Nerv, maxillaris superior verläuft, aufgesucht. Dann wird das Periost ebenso von der Orbitalfläche des großen Keilbeinflügels abgeschabt und dadurch das äußere Ende der Fissura orbitalis superior sichtbar gemacht. Man setzt hierauf den Meißel vertikal in der inneren Hälfte der Orbitalfläche des großen Keilbeinflügels auf und durchschlägt den Knochen von der Furche für den Nerv. maxillaris superior unten bis oben an das äußere Ende der Fissura orbitalis. Das Fenster, welches man im Knochen anlegt, wird nach außen noch $\mathrm{um} 1 / 2 \mathrm{~cm}$ erweitert, es muß den ganzen unteren Knochenrand der Orbitalfissur in sich begreiten und mit seinem unteren Rande in gleicher Höhe mit dem Boden der Orbita stehen. Jetzt liegt die Dura mater der mittleren. Schädelgrube frei, die am Leichnam wenigstens ohne Mühe etwa $1 \mathrm{~cm}$ weit von der Außenwand des Sinus cavernosus abgelöst werden kann. Zur Inzision des Sinus wird ein vorn abgestumpftes Messer mit der Schneide gegen ihn in der Höhe des Bodens der Orbita eingeführt und gegen den Keilbeinkörper vorgeschoben, bis es auf knöchernen Widerstand trifft. Der Schnitt fällt bei dieser Anlage stets unterhalb der im Sinus cavernosus verlaufenden Art. carotis interna. Am Lebenden wird bei Vorhandensein eines Thrombus oder von Eiter im Sinus letzterer wahrscheinlich nach Ablösung der Dura von seiner äußeren Wand spontan einreißen und dadurch die weitere Operation erleichtert werden. Man geht schließlich in die spontan entstandene oder künstlich im Sinus angelegte Öffnung mit einer kleinen Kürette ein und entfernt mit ihr den pathologischen Inhalt aus dem ganzen Lumen bis rückwärts zu den Einmündungsstellen des Sinus petrosus superior und inferior. Der Abstand vom äußeren Rande der Orbita beträgt im Durchschnitt $7 \frac{1}{2}$ bis $81 / 2 \mathrm{~cm}$. M. erwähnt, daß er auf die richtige Ausbildung dieses Verfahrens erst durch einen Mißerfolg am Lebenden gebracht worden ist, und daß er es dann an der Leiche zehnmal hat durchführen können.

\section{Diskussion.}

Barnhill (Indianopolis) hält die Operation für möglich. fragt den Vortr. aber, wie er bei den Vornahmen am Sinus das Hirn zur Seite halten will. Dieses dürfte an der Leiche einfacher gewesen sein als bei einem an Cavernosusthrombose Erkrankten, dessen Hirn sich jedenfalls in einem Zustande des Ödems befindet. 
Lutz (Brooklyn) fragt den Vortr., ob er die vor mehreren Jahren vorgeschlagene Bloßlegung des Sinus cavernosus auf intranasalem Wege, durch das Siebbein und Keilbein, versucht hat.

Reik (Baltimore) wünscht zu wissen, welche Vorzüge das beschriebene Operationsverfahren vor demjenigen von Ballance hat; bei welchem das Auge nicht geopfert wird.

Mc Cuen Smith (Philadelphia) betont die Schwierigkeiten, die sich dem sicheren Erkennen einer Cavernosusthrombose entgegenstellen.

Dench (New York) erinnert, daß Ballance gesagt hat, er würde das nächste Mal durch die Orbita eingehen. Vortr. hat mithin das von Ballance angedeutete Operationsverfahren ausgeführt. Die Preisgabe des Auges in solchen Fällen ist gerechtfertigt.

Day (Pittsburg) bemerkt, daB bei allen Cavernosusthrombosen, die er gesehen hat, beide Sinus ergiffen waren und beiderseitiger Exophthalmus vorhanden war.

Mosher (Schlußwort) hält das Abheben des Gehirns für nicht so schwierig. Das von ihm empfohlene Operationsverfahren scheint ihm nach seinen Versuchen an der Leiche der intranasalen Operation und derjenigen von Ballance überlegen zu sein. Er widerspricht entsohieden der Behauptung Days, daß die Thrombose sich regelmäßig auf beide Sinus cavernosi erstreckt. In seinem Falle fand sich nur Exophthalmus auf einer Seite und verhielt sich der andere Sinus cavernosus makroskopisch normal. Die Fortnahme des Auges ist hier gerade so erlaubt, wie bei bösartigen Erkrankungen des Oberkiefers und der Orbita. Ob danach eine sympathische Entzündung des zweiten Auges zu fürchten ist, worauf Dench hindeutete, weiß er nicht. In Fällen von Herausnahme des Bulbus wegen maligner Geschwülste hat er eine solche nicht beobachtet.

9. Bryant (New York): Bemerkungen über den Wert der Radiumbehandlung bei Ohrenkrankheiten. Die Wirkung, welche wir vom Radium in der Ototherapie verlangen, ist, daß es einmal das Wachsen normalen Gewebes veranlaßt, andererseits neugebildetes oder narbiges Gewebe zum Einschmelzen bringt. Beide Wirkungen können je nach der Natur des Falles einzeln oder vereint erwünscht sein. Br. verwendet als Radiumpräparat das Brom-Mesothorium, weil es die für therapeutische $Z$ wecke vornehmlich in Betracht kommenden $\beta$-Strahlen am reichlichsten ent- 
sendet; er bedient sich als Applikators einer Glasröhre, die $5 \mathrm{mg}$ in Stanniol eingewickeltes Brom-Mesothorium enthält, und die er in den äußeren Gehörgang einführt. Mit ganz besonderer Dringlichkeit wird auf die nötige Vorsicht bei der Radiumbehandlung hingewiesen, um vor allem zu verhüten, daß sie dem Kranken Schaden bringt. Die individuelle Empfindlichkeit dem Mittel gegenüber muß in jedem einzelnen Falle ausgeprobt werden, ebenso aber die Stärke der Wirkung jeden neuen Applikators, wenn er auch die gleiche Radiummenge, wie der bisher benützte, enthält. Als Kontraindikation der Behandlung wird allein totale Zerstörung des Labyrinths betrachtet, als Wirkungsgebiet gelten alle Arten von Funktionsstörung des Ohres, in erster Reihe stationäre und Endzustände. Eine auf die Ursachen der Hörstörung gerichtete Therapie wird zweckmäßig vorausgeschickt, ferner empfiehlt es sich, die Radiumbehandlung mit anderen therapeutischen Maßnahmen zu verbinden, für deren Einwirkung sie das $\mathrm{Ohr}$ manchmal empfänglicher macht. Br. berichtet kurz über 20 Fälle aus seiner Privatpraxis, die von ihm schon jahrelang zuvor beobachtet worden waren, und bei denen er das Radium in Anwendung gebracht hat. Die Ergebnisse lassen sich, nach der Anzahl der Ohren berechnet, folgendermaßen zusammenstellen:

Hypertrophischer Mittelohrkatarrh: Gehör normal geworden 2, wesentlich gebessert 2.

Atrophischer Mittelohrkatarrh: Gehör normal geworden 4 , wesentlich gebessert 6 , unverändert 2 .

Otosklerose: Gehör wesentlich gebessert 3, wenig gebessert 6 .

Residuen chronischer Mittelohreiterung: Gehör wesentlich gebessert 3 , wenig gebessert 7 , unverändert 4 .

Nichteitrige Labyrinthitis: Gehör wesentlich gebessert, 1, verschlechtert 1 .

Von 40 behandelten Ohren sind mithin in bezug auf das Gehör 20 normal oder bedeutend besser geworden.

\section{Diskussion.}

(rockett (Boston) hat unter 8 mit Radium behandelten Otosklerosefällen nur dreimal einige Besserung des Gehörs gesehen, die Ohrgeräusche wurden vorübergehend gelindert. Er glaubt im Gegensatz zum Vortr. nicht, daß sich durch Radium Dauererfolge werden erzielen lassen.

Day (Pittsburg) äußert sich in ähnlicher Weise skeptisch. Nur zweimal wurde eine Zunahme des Gehörs beobachtet, 
aber selbst in dem anscheinend am günstigsten beeinflußten Falle war sie nach einem Monat vollständig wieder zurückgegangen, die Patientin hatte zwar das subjektive Gefühl des Besserhörens, die Hörprüfung ergab aber dasselbe Resultat wie vor der Behandlung. Die subjektiven Geräusche scheinen vorübergehend zum Aufhören gebracht werden zu können. D. hat auf Grund dessen seine Versuche mit Radium nicht weiter fortgesetzt.

Jack (Boston) vermag überhaupt nicht einzusehen, wie das Radium bei einer knöchernen Fixation des Steigbügels, die in den Fällen von Otosklerose vorliegt, Nutzen bringen soll.

Bryant (Schlußwort) wiederholt, daß sich die Wirkung des Radiums nicht auf progressive Fälle erstreckt, sondern nur auf solehe, die zum Stillstand gekommen sind, oder in denen die Ursache der Erksankung beseitigt werden kann. In genügender Dosis angewandt, ist das Radium seiner Meinung nach auch imstande, Knochengewebe anzugreifen.

10. Randall (Philadelphia): Verschiedene Arten otogener Facialislähmung. R. erwähnt eine Reihe eigener Beobachtungen mit verschiedener Entstehung: Fortpflanzung einer Neuritis von der Paukenhöhle her nach außen, Druck vereiterter Drüsen vor dem Ohre, Verletzung des Nerven in der Paukenhöhle durch einen Schneckensequester, bei der Chromsäureätzung cines Polypenstumpfes, bei der Extraktion eines den Nerven umschlieBenden Cholesteatoms, Verletzung wahrscheinlich am Hiatus Fallopii beim Abkratzen des Tegmen in einem Falle von ExtraduralabszeB und in die Schädelhöhle hineingewachsenem Cholesteatom, Lähruung des Nerven bei Herpes auricularis. Mit Rücksicht auf die Behandlung warnt er vor der Anwendung der Elektrizität, selbst als konstanter Strom, solange noch Zeichen von Neuritis bestehen; er glaubt im übrigen, daß sie durch mildere Mittel ersetzt werden kann. Das Gleiche gilt von der Nerven-Anostomose, welche R. durchaus absprechend beurteilt. R. legt das Hauptgewicht auf alterierende und absorbierende Mittel, im Beginn bei noch vorkandenen akut entzündlichen Erscheinungen Jod- und Brompräparate, später eine Arsen und Jodquecksilber enthaltende Mischung, sowie ferner auf eine Massage der Ge sichtsmuskeln, die ohne Schaden von Anfang an und mehrmals täglich vorgenommen werden kann. Zum Beweis wird die schnelle Heilung einer durch einen Schlag auf den Kopf erzeugten und mit Haematotympanum verbundenen Facialislähmung durch Jodbehandlung mitgeteilt, während in einem 
zweiten Falle noch nach 4 Jahren unter fortgesetzter Massage eine bedeutende Besserung der nach der Radikaloperation aufgetretenen Lähmung erfolgte.

\section{Diskussion.}

Dench (New York), Lewis (New York) und Richardson (Washington) erwähnen Fälle von Facialislähmung, die nach der Mastoidoperation, der Kauterisation von Granulationsgewebe in der Paukenhöhle, dem Kürettement eines Extraduralabszesses entstanden war und durch. den elektrischen Strom zur Heilung gelangte.

Passemore (New York) hat in einem Falle von doppelseitiger Mikrotie dem Versuch der Anlegung eines äußeren Gehörganges eine komplette Facialislähmung auf der einen Seite folgen sehen. Diese verlor sich innerhalb weniger Monate unter einer von der Kinderfrau vorgenommenen Massage.

Barnhill (Indianopolis) bemerkt, daß die Zeit, bis zu welcher die Muskeltätigkeit sich wiederherstellt, von der Art der Verletzung abhängt and geringer ist bei unversehrtem als bei mitverletztem knöchernem Kanal. Die Schnelligkeit, mit der bei Anwendung der Elektrizität die Funktion zurückkehrte, war oft überraschend.

Me Kimmie (Washington) hält das Auftreten von Facialislähmung bei einer Mittelohreiterung auch, wenn keine Mastoidsymptome vorliegen, für ausreichend, um eine Anzeige zur Aufmeißelung abzugeben. In der Beobachtung Mc Ks. war die Lähmung 10 Tage nach der Operation verschwunden.

11. Shambaugh (Chicago). Die chirurgische Anatomie des Schläfenbeines. Vortr. demonstriert einige aus einer Reihe von 50 Schnitten durch das Schläfenbein, die er zu Lehrzwecken angefertigt hat. Er glaubt, daB der Studierende sich am sichersten die in Betracht kommenden Verhältnisse einprägen wird, wenn er täglich 2 bis 3 Stunden dazu verwendet, um diese Schnitte sorgfältig abzuzeichnen und die verschiedenen anatomischen Gebilde an jedem von ihnen mit ihren Namen zu versehen. Nach 6 bis 8 Wochen solcher Beschäftigung pflegt ein festhaftendes geistiges Bild wenigstens der wichtigsten Punkte aus der Anatomie des Ohres erworben zu sein.

\section{Diskussion.}

Mosher (Boston) verfährt beim Unterricht in der Anatomie der Nase und des Rachens in ähnlicher Weise wie der Vortr. 
Nur erleichtert er dem vielleicht im Zeichnen ungeübten Studenten die Arbeit dadurch, daß er das Präparat unter eine Glasplatte legen und auf dieser die allgemeinen Umrisse durchzeichnen läßt. Die Einzelheiten müssen aus freier Hand eingetragen werden.

Dench (New York) geht des näheren auf die nicht so selten vorhandene tiefe Zelle hinter der Fossa digastrica ein, die von den anderen, überliegenden Zellen durch eine dünne Knochenschicht getrennt wird und mit dem scharfen Löffel leicht nach Entfernung der Zellen an der Spitze des Warzenfortsatzes erreicht werden kann. D. hat in einem Falle bei Fortnahme der unteren knöchernen Gehörgangswand mit dem Meißel den Bulbus jugularis eröffnet; die Blutung wurde durch eine kleine Gazekompresse gestillt, der betreffende Teil der Wundhöhle wurde mittels Jodoformgaze isoliert, und 10 Tage später wurde die Hauttransplantation vorgenommen. Sodann erwähnt $\mathrm{D}$., daß er folgende Merkzeichen anzugeben pflegt, um direkt den Aditus und das Antrum zu erreichen. Wenn man eine vertikale Tangente an die hintere und eine horizontale Tangente an die obere Wand des knöchernen Gehörganges legt, so entsteht ein Dreieck, dessen Spitze durch den Schnittpunkt der beiden Tangenten und dessen Basis durch den zwischen ihnen befindlichen Teil der hinteren oberen Gehörgangswand gebildet wird. Dieses Dreieck ist unmittelbar über dem Aditus ad antrum gelegen.

Randall (Philadelphia) hält Photographien für weit instruktiver als gezeichnete Abbildungen. Er exklärt es ferner für wichtig, auch entwicklungsgeschichtliche Tatsachen zu lehren, so, daß das membranöse Labyxinth vor dem knöchernen vorhanden ist, daß die Entstehung des Nervus facialis der seines Knochenkanals vorhergeht, und daß überhaupt die knöchernen Teile sich erst um die Weichgebilde herum entwickeln, diese mithin das Konstante, jene das Variierende sind. Außerdem macht R. auf die Zellen längs des inneren Gehörganges aufmerksam, die nach seiner Erfahrung, versehieden stark ausgebildet, sich immer vorfinden. Sie liegen an der oberen. Wand oder auch an anderen Wänden des Meatus internus, erstrecken sich manchmal bis in die Sella turcica und sind oft lufthaltig.

Phillips (New York) geht bei der Aufsuchung des Antrum immer von der Spina supra meatum aus, die in einzelnen Fällen zwar fehlt, gewöhnlich aber ihrem Orte nach bestimmt werden kann. 
Mosher bemerkt, daß die Linea temporalis nur in etwa einem Drittel der Fälle die untere Grenze der mittlexen Schädelgrube bezeichnet. Bei zwei Dritteln liegt die Basis des Gehirns oberhalb dieser Linie.

Rae (New York) führt aus, daß die Bestimmung sowohl des von Macewen als des von Dench angegebenen Dreiecks als Merkzeichen für die Lage des Antrum Schwierigkeiten machen und leicht durch folgende Methode ersetzt werden kann. Der vordereWundrand wird soweit zurückgezogen, daß die bogenförmige hintere obere Umgrenzung des knöchernen Gehörganges frei übersichtlich ist. Von ihr aus als Basis konstruiert man ein gleichseitiges Dreieck, und dieses gibt das gewünschte Merkzeichen ab.

12. Phillips (New York): Ein weiterer Bericht über Fälle von entzündlichen Labyrintherkrankungen. Vortr. teilt, bis auf die letzte ohne weiteren Kommentar, folgende Beobachtungen mit. a) 52 jähriger Mann mit rechtsseitiger chronischer Mittelohreiterung und Cholesteatom in Paukenhöhle und Antrum. Vor 8 Wochen stellten sich Anfälle von heftigem Schwindel, Übelkeit, Erbrechen und Schmerzen in der rechten Kopfhälfte ein. In den letzten beiden Wochen Befinden besser. Rechts totale Taubheit und vestibulare Unerregbarkeit. Kein Fistelsymptom. Totalaufmeißelung und Dränage des Labyrinths. Große Fistel im horizontalen Bogengang, ovales Fenster offen. Nach der Operation beinahe $2 \mathrm{Mo}$ nate lang frei von Beschwerden bis auf die Kopfschmerzen. Daun meningitische Symptome, Entleerung eines großen Schläfenlappenabszesses, Tod nach 24 Stunden. Keine Sektion. - b) 24 jähriger Mann mit chronischer Otitis media purulenta dextra. In den letzten Wochen Zeichen von Labyrinthreizung. Gehör erhalten, vestibulare Erregbarkeit (Rotation) eher gesteigert, positives Fistelsymptom. Totalaufmeißelung, große Bogengangfistel. Geheilt mit erhaltener Cochlear- und Vestibularfunktion, aber zurückbleibender Facialislähmung. - c) 10jähriges Mädchen. Rechts chronische Eiterung, totale Taubheit, kalorische Reaktion vorhanden, Fistelprobe negativ. Totalaufmeißelung, keine Labyrinthfistel. Wundhöhle noch nicht epidermisiert. (Dr. Shar p.) - d) Patient war wegen seiner rechtsseitigen Mittelohreiterung schon zweimal, im 8. und 16. Lebensjahre (das letzte Mal radikal), operiert worden. Trotzdem Eiterung fortdauernd. In den letzten Tagen leichte Kopfschmerzen. Wundhöhle nur zum Teil epidermisiert, an der inneren Paukenhöhlenwand einige Granulationen. Totale Taubheit, kalorische 
Reaktion fehlte. Bei der nochmals wiederholten Eröffnung der Mittelohrräume wurden beide Fenster und überhaupt die ganze Promontorialwand mit dichtem neugebildetem Knochen bedeckt gefunden. Heilung der Eiterung. - e) Patientin 18 Jahre alt. Nach 6 wöchiger Dauer einer akuten Otitis media purulenta sinistra Auftreten von Schwindel, zuerst nur bei Drehungen des Kopfes, dann auch im Liegen. Ebenso erst in den letzten Tagen Übelkeit bzw. Erbrechen und leichter Nystagmus zur gesunden Seite. Laute Sprache mit dem kranken Ohre gehört. Fistelsymptom vorhanden. Totalaufmeißelung. Großer perisinuöser Abszeß. Große, mit einem Granulationspolster bedeckte Fistel an der Konvexität des horizontalen Bogenganges. Nach der Operation Schwindel nicht wiedergekehrt, während der Nystagmus nach rechts bei Blick nach rechts noch 2 Tage lang fortbestand. Geheilt mit einem Gehör von $20^{\prime}$ für Flüstern. (Dr. Thomson.) f) Männlich. Beiderseitige chronische Mittelohreiterung, Schwindel seit 14 Tagen, starker Kopfschmerz. Rechts nur Spuren von Gehör, kalorische Probe negativ. Temperatur 37,7 bis $39^{\circ}$, Pulsfrequenz 60 bis 88 . Spontannystagmus nach links. Wegen früherer Syphilis Lumbalpunktion: im Liquor reichlich Streptococcus mucosus, Milchsäure im Überschuß, Fehlen der Kupfer reduzierenden Substanz. Nach vorübergehender Besserung der Symptome erneute Verschlimmerung mit Bewußtlosigkeit und Lähmung des rechten Facialis. Operation für Dränage der Cisterna magna, wobei sich nur wenig Flüssigkeit entleerte. Tod in der folgenden Nacht. Sektionsbefund: Alte Verdickungen der Meningen und daneben eine frische Meningitis purulenta, besonders an der Basis und hier am stärksten in der hinteren Schädelgrube. Eine andere, mehr abgegrenzte Eiteransammlung am rechten inneren Gehörgang und in ihm den Akustikus und Facialis umspülend. Knochen der inneren Seite des rechten Felsenbeins, einschließlich des Tegmen tympani et antri, dunkel verfärbt. Paukenhöhle und Warzenzellen mit Eiter gefüllt. Hammer intakt, Amboß fehlte; über die Beschaffenheit der Fenster und den Fortleitungsweg auf das innere Ohr wird nichts gesagt, außer, daß das Promontorium intakt war. Schneckeneiterung, in den inneren Gehörgang durchgebrochen.

\section{Diskussion.}

Dench (New York) berichtet im Anschluß an die letzte Beobachtung des Vortr. über einen Fall, in dem desgleichen 
früher Syphilis vorhanden gewesen war, das Mittelohr eiterte und das Labyrinth sich als funktionslos erwies. Es handelte sich um eine Perilabyrinthitis. Nach Ausführung der Radikaloperation erholte sich das Labyrinth wieder, und die Hörtätigkeit kehrte zurück (Flüstern 23'). D. erklärt sich als entschiedener Gegner eines zu aktiven Vorgehens dem Labyrinth gegenüber, da der Exstirpation dieses oft tödliche Meningitis nachfolgt. Er beschränkt sich auf die Entfernung des erweichten Knochens ringsum die Fistel und wartet dann $a b$; ein in solcher Weise von seinen Labyrinthsymptomen befreiter Fall wird mitgeteilt.

Shambaugh (Chicago) macht auf die Fälle von Neuritis des Hörnerven bei Fehlen einer Mittelohreiterung aufmerksam, in denen je nachdem allein der Ramus vestibularis oder cochlearis oder beide zugleich ergriffen sind, entweder nur Schwindel oder nur Schwerhörigkeit und Ohrensausen vorhanden sind oder die genannten Störungen sich miteinander vergesellschaften. Diese Neuritis acustica ist oft eine toxische, und der Ursprung der Infektion ist in nicht wenigen Fällen in den Tonsillen $z u$ suchen.

Randall (Philadelphia) hat sowohl Labyrinth-als Ponssymptome allein unter medikamentöser Behandlung verschwinden sehen und ist auf Grund dessen mit operativen Eingriffen sehr zurückhaltend. Die Flut der Begeisterung für die Labyrintheröffnung scheint ihm schon wieder wesentlich abzuebben. Bei einer Patientin mit Erscheinungen, die auf den Kleinhirnbrückenwinkel hindeuteten und nach Influenza. aufgetreten waren, wurde bei der intrakraniellen Operation eine Perineuritis mit so fester Verlötung des Akustikus und Facialis gefunden, daß der Chirurg mehr als eine halbeStunde brauchte, um beide Nerven voneinander zu trennen. Zur. Dränage bei Meningitis erachtet $R$. das Eingehen durch die Schläfenbeinschuppe für wirksamer als die Dränage der Cisterna magna.

Phillips (Schlußwort) stimmt der Wichtigkeit einer sorgfältigen Untersuchung der Labyrinthfunktionen in jedem Falle von Mittelohreiterung zu. In akuten Fällen hält er es desgleichen für besser, das Labyrinth nicht anzugreifen; andererseits aber ist die Zeit immer nur kurz, während welcher man von einer Labyrinthoperation noch etwas erhoffen kann. Wenn sich erst eine Meningitis entwickelt hat, wird jede Operation zu spät kommen.

13. Holmes (Boston): Ohrkomplikationen bei TyArchiv f. Ohrenheilkunde. Bd, 97. Otolog. Rundschau. 
phus. In Fällen von Typhus ist es von größter Bedeutung, daß dem Zusțande des Gehörorgans stets die volle Aufmerksamkeit des behandelnden Arztes zugewandt wird, da der Typhus sowohl an sich Ohrenleiden hervorrufen als bei bereits vorhandenen eine Verschlimmerung bewirken kann. Andererseits können durch Übergreifen einer Ohreiterung auf den Sinus transversus und die Vena jugularis typhusähnliche Symptome hervorgebracht werden, ohne daß Typhus besteht, und schließlich ist die Möglichkeit gegeben, daß im Verlaufe eines Typhus eine frisch entstandene oder schon ältere, exazerbierte Otitis eine Sinus- und Jugularisthrombose veranlaßt und dadurch das Krankheitsbild im höchsten Grade kompliziert. Vortr. geht besonders auf die letzterwähnten beiden Arten von Fällen ein, deren richtige Auffassung deshalb so schwierig ist, weil wegen des schwer beeinträchtigten Allgemeinbefindens eine genaue Anamnese sich nicht erlangen läßt, Ohrschmerzen, Druckempfindlichkeit oder Schwellung in der Umgebung des Ohres, Empfindlichkeit längs des Verlaufes der Jugularvene, Veränderungen des Augenhintergrundes möglicherweise fehlen, ja selbst das Trommelfellbild manchmal keine charakteristischen Veränderungen zeigt. Täuschungen werden außerdem dadurch noch erleichtert, daß der Typhus selbst unter Umständen septische Symptome hervorbringt und Rückfälle mit erneutem Ansteigen der Temperatur zu machen imstande ist. Wenn sich gar schon eine konsekutive Meningitis entwickelt hat oder metastatische Herde in den verschiedensten Organen sich vorfinden, werden die Zeichen der Ohrerkrankung meist vollständig verdeckt, In solchen Fällen kann es nur durch sorgfältigste Beachtung aller vorhandenen Symptome, genaue Lokaluntersuchung und Ausschluß aller sonstigen Möglichkeiten gelingen, die richtige Diagnose auf Ausgang der septischen Symptome vom Gehörorgan bzw. Sinus transversus zu stellen und damit den Weg für die allein lebensrettende Therapie zu finden. Vortr. legt in diagnostischer Beziehung Wert auf eine vergleichende Palpation der Jugulares beider Seiten, bei welcher sich nicht selten eine Empfindlichkeit auf der Seite der Erkrankung nachweisen läßt. Die Halsmuskeln, besonders die Kopfnicker, müssen dabei erschlafft sein, auf etwa vorhandene sehmerzhafte Lymphdrüsen ist zu achten, ein negatives Resultat beweist nichts gegen Thrombose. Weit weniger Bedeutung hat die Blutuntersuchung, da Leukocytose häufig, und selbst in den schwersten Fällen, fehlt. Vortr. berichtet im Anschluß 
über fünf von $\mathrm{ihm}$ in den letzten vier Monaten operierte Kranke mit Sinusthrombose, bei denen die Symptome an Typhus erinnerten und solcher auch viermal fälschlich von den früher behandelnden Ärzten angenommen worden war. Daher konnte die Operation erst verhältnismäßig spät gemacht werden. Zwei Kranke wurden geheilt, drei gingen an Meningitis zugrunde, die zweimal wenigstens sicher schon vor dem chirurgischen Eingriff bestanden hatte.

\section{Diskussion.}

Randall (Philadelphia) ist der Ansicht, daß otitische Sinusthrombose als Komplikation des Typhus sehr selten ist.

Day (Pittsburg) erwähnt, daß gelegentlich einer Epidemie vor mehreren Jahren er und Dr. Jackson über 800 Typhusfälle beobachtet haben, von denen 5 Prozent Ohrkomplikationen zeigten. Diese traten gewöhnlich zu Anfang der vierten Woche auf. Sinusthrombose wurde nicht ein einziges Mal gesehen. Wenn bei einem Typhuskranken mit hohem Fieber und Otitis das Fußende des Bettes höher gestellt wurde, bekam er fast sicher eine Mastoiditis. Die Ohrkomplikationen des Typhus beruhen auf der herabgesetzten Vitalität der Gewebe, die den Mikroorganismen ihre Einwirkung erleichtern.

Holmes (Schlußwort) fügt seinen Mitteilungen hinzu, daß unter den von ihm beobachteten Typhuskranken 3 bis 4 Prozent Schwerhörigkeit durch toxische Neuritis des Akustikus hatten, die oft noch wochenlang fortdauerte, nachdem das Sensorium schon wieder frei geworden war. Sinusthrombose bei Typhus hält er für nicht so selten, er hat sie bei 5 bis 6 Prozent der Typhusfälle im Jahre gesehen. In den oben erwähnten fünf Beobachtungen war nur einmal Typhus wirklich vorhanden, bei den vier anderen täuschte das durch die Sinusthrombose hervorgerufene Bild nur einen solchen vor.

14. Page (New York): Angeborene beiderseitige Mikrotie mit totalem knöchernem Verschluß des äußeren Gehörganges, Operation und Bericht von Fällen. Die Gründe, die gegen den Versuch der 'Anlegung eines künstlichen Gehörganges in Fällen von angeborener totaler knöcherner Atresie geltend gemacht worden sind, hält P. nicht für stichhaltig, da die Erfahrung zeigt, daß bei sonst nicht mißgebildeten, lebensfähigen Kindern trotz der Entwicklungsstörung in den äußeren Teilen das Labyrinth 
und wohl auch der Steigbügel stets intakt sind, eine Feststellung, ob Gehör vorhanden ist, ohne Mühe gemacht werden kann und bei richtigem Operationsverfahren ein Wiederverschluß des geschaffenen Kanals sich verhüten läßt. P. berichtet über acht meist mit günstigem Resultat operierte Fälle, von denen vier der Literatur entnommen sind, vier dem Manhattan Eye, Ear and Throat Hospital entstammen und noch nicht veröffentlicht worden sind. Darunter befindet sich ein von $P$. selbst im Alter von 2 und 4 Jáhren auf beiden Seiten operiertes Kind, bei dem ein für das gewöhnliche Leben völlig ausreichendes Gehör erzielt wurde und der Gehörgang des erstoperierten Ohres noch 2 Jahre später einen Durchmesser von $5 \mathrm{~mm}$ hatte. Der Eingriff soll, um dem Kinde durch die Hörverbesserung eine leichtere Geistes- und Sprachentwicklung zu ermöglichen, wenn es angeht, bei Ablauf des zweiten Lebensjahres gemacht werden, vorausgesetzt, daß das Kind sonst wohlgebildet ist und auf ihm durch die Luft zugeführte Töne oder Geräusche, wie Pfeifen, Händeklatschen, Rufen usw., reagiert. Zuvor empfiehlt es sich, durch eine Röntgenaufnahme (unter Anästhesie) sich möglichsten Aufschluß über die vorliegenden anatomischen Verhältnisse zu verschaffen. Die Operation besteht in der Aufmeißelung des Antrum und daran sich anschließender Fortnahme des Knochens, der: sich nach vorn von ihm an Stelle des Gehörganges vorfindet. Eine Verletzung des oft der vorderen Antrumwand anliegenden Unterkiefergelenkes wird dadurch vermieden, daß man den Knochen vorsichtig von hinten nach vorn abträgt. Wofern nicht ein normales Trommelfell vorhanden ist, wird aucb der gesamte Paukenhöhleninhalt bis auf den Steigbügel ausgeräumt, so daß die Labyrinthwand breit freiliegt, und wird außerdem das Ostium tympanioum tubae, wenn ein solches da ist, durch Kürettieren zum Verschluß gebracht. Alsdann spaltet man die rudimentäre Ohrmuschel durch einen Kreuzschnitt, schlägt die so gebildeten vier Lappen nach innen und fixiert sie daselbst, damit ein genügend weiter Gehörgang entsteht, und vernäht schließlich die retroaurikuläre Wunde primär. Um eine schnelle Epidermisierung der Operationshöhle zu erzielen, soll nach 8 bis 10 Tagen eine Thierschsche Hauttransplantation vorgenommen und, wenn aus irgendeinem Grunde die Läppchen nicht haften, dieser Versuch sofort noch ein zweites und nötigenfalls ein drittes Mal wiederholt werden. 


\section{Diskussion.}

Berens (New York) bemerkt zu seinem vom Vortr. angeführten (noch nicht veröffentlichten) Falle, daß das fünf Jahre alte Kind vor der Operation auf beiden Seiten völlig taub erschien, da es weder auf Geräusche noch auf Stimmgabeltöne in Luft- oder Knochenleitung reagierte. Wenige Monate nach der Operation fing das Kind an, mit den anderen im gleichen Saale befindlichen Kindern zu sprechen, und der zufällige Beobachter konnte keine Hörstörung mehr an ihm nachweisen. Es kam B. bei dem von ihm gemachten, eine Art Radikaloperation darstellenden Eingriff nicht darauf an, einen tiefen Kanal herzustellen, sondern er legte sozusagen eine napfförmige Öffnung an mit dem Mittelohr am Grunde des Napfes.

Randall (Philadelphia) hat in ähnlichen Fällen dadurch eine bedeutende Hörverbesserung erzielt, daß er die Ohrtrompete frei durchgängig machte, und er rät deshalb, hierfür immer zu sorgen. Bei den von ihm operierten Kranken waren die Resultate sehr zufriedenstellend, wenn es gelang, den angelegten Kanal weit offen zu halten; anderenfalls ließ der Erfolg zu wünschen übrig. Zur Bekämpfung einer narbigen Verengerung empfiehlt R. das Einführen eines Gummirohrs oder einer aus einer dünnen Bleiplatte, wie sie sich in den Teekisten vorfindet, zusammengewickelten Rolle, die nach Bedarf allmählich weiter gemacht werden kann und doch fest genug bleibt, um der Kontraktion der Gewebe Widerstand zu leisten.

15. Perkins (New York): Bericht über Fälle von Infektion des Ohres mit dem Streptoceocus mucosus. Die 8 mitgeteilten Beobachtungen bestätigen die bekannte Tatsache, daß die Mucosus-Otitis zwar auch unter dem Bilde einer gewöhnlichen akuten eitrigen Mittelohrentzündung verlaufen und ohne Komplikationen zur Heilung gelangen kann, daß aber bei der überwiegenden Mehrzahl die Infektion sich auf den Knochen des Warzenteils ausbreitet und in ihm mehr weniger umfangreiche Zerstörungen hervorruft (fünfmal perisinuöser Abszeß, einmal Sinus bloßliegend), und zwar oft, ohne daß die Entzündung in der Paukenhöhle besonders hervorgetreten ist, bei einem bis auf Trübung und Fehlen des Oberflächenglanzes nur wenig veränderten, manchmal imperforiert gebliebenen Trommelfell. In einem Falle stellte sich beinahe 4 Monate nach der Mastoidoperation, als 
die Wunde hinter dem Ohre fast verheilt war, plötzlich eine rasch zum Tode führende Meningitis ein. P. zieht aus seinen Beobachtungen folgende Schlüsse: a) Der Streptococcus mucosus scheint eine besondere Affinität zum Knochengewebe zu haben. b) Wenn eine breite Verbindung zwischen Paulkenhöhle und Antrum besteht, kann durch Dränage der ersteren mittels einer frühzeitigen und breiten Trommelfellinzision Heilung herbeigeführt werden. c) Für die Abschätzung des Verhaltens des Warzenfortsatzes und damit für die Anzeige zur Mastoidoperation kann das Röntgenverfahren von großer Bedeutung sein. d) Die AufmeiBelung muß möglichst früh und ausgiebig gemacht werden, sobald man sich von dem Vorhandensein unausgleichbarer Veränderungen im Warzenknochen überzeugt hat. e) Die betreffenden Fälle müssen, ob sie operiert worden sind oder nicht, stets längere Zeit sorgfältig überwacht werden, da Spätkomplikationen hier verhältnismäBig häufig sind. f) Kranke im höheren Lebensalter und Diabetiker scheinen zur Infektion mit dem Streptococcus mucosus mehr als zu der mit anderen Mikroorganismen hinzuneigen.

\section{Diskussion.}

Dench (New York) spricht sich bei aller Anerkennung des Ernstes einer Mucosus-Otitis dahin aus, daß ein großer Prozentsatz der Fälle doch ohne Operation am Warzenfortsatz geheilt wird. Unter 13 Kranken seiner Privatpraxis hat er nur bei 2 die Aufmeißelung machen müssen. Daß die Röntgenphotographie unter Umständen irreführen kann, beweist eine eigene Beobachtung, bei der das wolkige Aussehen des Warzenfortsatzes wahrscheinlich von früher überstandenen Entzündungen herrührte. Es wurde nur die Parazentese gemacht, und 5 Wochen danach war Genesung eingetreten.

16. Wilson und Pike: Die Beziehungen des Labyrinths zum Kleinhirn und Großhirn. W. legt, zum Teil auf frühere Arbeiten der beiden genannten zurückgreifend, zunächst die Gründe dar, die für eine Trennung der langsamen und schnellen Komponente des otogenen Nystagmus nach Ursache und Auslösungsart sprechen. Sodann berichtet er zunächst über von ihm gemeinsam mit Pike angestellte Versuche mit mechanischer, kalorischer and elektrischer Reizung des Labyrinths bei Hunden. Um gleichlautende Resultate zu erhalten, müssen die Versuche immer in der- 
selben Lage des Tieres und mit derselben Methode der Eröffnung des Labyrinths vorgenommen werden. Die anästhesierten Tiere zeigten nur eine Seitwärtsstellung der Augen, die je nach der gereizten Seite und dem verwendeten Reize verschieden war, und zwar ergaben heißes Wasser und der negative Pol einerseits und kaltes Wasser und der positive Pol andererseits je eine gleichsinnige Ablenkung, die im ersteren Falle entgegengesetzt gerichtet wie im letzteren war. Gleich starke elektrische Reizung beider Seiten brachte die Augen nicht aus ihrer Ruhestellung, bei ungleich starker Reizung bewegten sich die Augen nach der dem stärkeren Reize entgegengesetzten Seite. Die Kathode stellte der Anode gegenüber den stärkeren Reiz dar. - Eine zweite Versuchsreihe beschäftigte sich mit den Wirkungen der Labyrinthzerstörung. Nach einseitiger Zerstörung lieB sich eine $A b-$ lenkung der Augen nach der verletzten Seite und während der ersten Tage außerdem ein nahezu vertikaler Nystagmus an dem Auge der operierten Seite und ein horizontaler Nystagmus an dem Auge der nicht operierten Seite feststellen. Ferner zeigten die Tiere eine Verdrehung des Kopfes nach der. Seite der Verletzung und waren nicht imstande, auf ihren Füßen zu stehen oder zu gehen, ohne nach der verletzten Seite umzufallen. Diese Gleichgewichtsstörungen verminderten sich in der Folge und waren am Ende der zweiten Woche verschwunden, während die Verdrehung des Kopfes nach der operierten Seite mit dem Hinterhaupt nach unten und vorn und eine gleichgerichtete leichte Neigung der vorderen Körperhälfte anhielten. Wurde bei demselben Tiere später auch noch das zweite Labyrinth zerstört, so waren die hiernach auftretenden Störungen um so geringer, je größer die Zwischenzeit zwischen den beiden Eingriffen war. Eine Verdrehung des Kopfes und der vorderen Körperhälfte nach der zuletzt operierten Seite machte sich nicht bemerkbar, vielmehr kehrten beide nahezu ganz in ihre normale Stellung zurück. Der Nystagmus dauerte nur wenige Stunden und war am nächsten Tage wieder verschwunden. - Versuche mit teilweiser oder totaler Entfernung des Kleinhirns legten auf das deutlichste dar, daß sowohl die Ablenkung der Augen als der Nystagmus nach Reizung oder Zerstörung des Labyrinths vom Kleinhirn vollständig unabhängig sind, und daß die Bahn vom Labyrinth zu den Augenmuskeln daher außerhalb des Kleinhirns verläuft. Der Nystagmus und das übrige Verhalten der Tiere nach Labyrinth-, and nach Kleinhirn- 
verletzung sind von Grund aus verschieden. - Um die Frage des Verlaufes der Labyrinth-Augenbahn der Lösung näher zu bringen, wurden des weiteren verschiedene Teile des Großhirns fortgenommen, und zwar mit folgenden Ergebnissen: Die Exstirpation des ganzen Großhirns und der Thalami, so daB nichts als Pons, Medulla oblongata und ihre Verbindung mit den Augennerven zurückblieb, hatte keine Änderung in der nach Reizung oder Zerstörung des Labyrinths auftretenden Ablenkung der Augen, d. i. der langsamen Komponente des Nystagmus, zur Folge. Diese war vielmehr allein auf das Erhaltensein des hinteren Longitudinalbündels angewiesen. Im Gegensatz hierzu wurde die schnelle Komponente des Nystagmus durch die Entfernung der Großhirnrinde beeinflußt, so daß sie bei enthirnten Tieren, einschließlich der Thalami optici, aber mit erhaltener Okulo-Vestibularbahn, fehlte. Speziell traten in den von W. und P. angestellten Versuchen die betreffenden Änderungen bei Verletzung der Rinde des Sohläfenlappens und der angrenzenden Teile hervor. Reizung des Labyrinths auf der der Verletzung entgegengesetzten Seite durch kaltes Wasser oder die Anode ergab unter diesen Umständen einen typischen labyrinthären $\mathrm{Ny}^{-}$ stagmus, Reizung durch heißes Wasser oder die Kathode nur seitliche Ablenkung, aber keinen Nystagmus. Ebenso war Zerstörung des Labyrinths auf der verletzten Seite nicht von Nystagmus gefolgt. Mehrmals wurde beobachtet, daß das Auge der verletzten Seite eine Drehbewegung um eine sagittale Achse zeigte, das andere Auge aber von einer solchen freiblieb.

W. und P. stellen folgende Theorie über den Mechanismus des Nystagmus auf. Durch einen Antrieb, der beim labyrinthären Nystagmus im Labyrinth liegt, wird zuerst eine Ablenkung der Augen aus ihrer Gleichgewichtsstellung veranlaßt. Dadurch werden der Musc. rectus internus oculi auf der Seite der Ablenkung nach außen und der Muse. rectus externus auf der anderen Seite in stärkere Dehnung versetzt, und der hierdurch erzeugte Reiz wird durch Vermittlung der dritten, vierten und sechsten Hirnnerven auf das Cerebrum übertragen. Die zentripetalen Impulse lösen dann in den okulomotorischen Zellen des Großhirns zentrifugale Impulse aus, die eine schnelle Kontraktion der gedehnten Augenmuskeln mit Erschlaffung ihrer Antagonisten bewirken. So kehren die Augen mit einer schnellen Ruckbewegung in ihre Medianstellung zurück, womit bei gesundem Gehirn 
die Muskelwirkung aufhört. Verletzungen der Großhirnhemisphäre auf der Seite der langsamen Deviation der Augen heben die schnelle Komponente des Nystagmus ganz oder teilweise auf, weil -von der Hemisphäre dieser Seite die Impulse ausgehen, die die Augen in ihre Ruhestellung zurückführen.

Zahlreiche Demonstrationen am Projektionsapparat erläutern die mitgeteilten Versuche und ihre Resultate.

Blau. 